\title{
Origin of Excitation Dependent Fluorescence in Carbon Nanodots
}

\author{
Arjun Sharma, ${ }^{\dagger}$ Trilochan Gadly, ${ }^{\ddagger}$ Alka Gupta, ${ }^{\S}$ Anand Ballal, ${ }^{\S}$ Sunil Kumar Ghosh ${ }^{\ddagger}$ and Manoj \\ Kumbhakar ${ }^{\dagger, *}$ \\ ${ }^{\dagger}$ Radiation \& Photochemistry Division, ${ }^{*}$ Bio-organic Division, ${ }^{\S}$ Molecular Biology Division, Bhabha \\ Atomic Research Center, Mumbai 400085, India
}

Email: manojk@barc.gov.in

\section{Material Synthesis}

Commercially available citric acid, diaminopropane and urea were used without any purification for the synthesis of CNDs. Milli-Q pure water was obtained from Milli-Q System (Millipore) and used in all synthetic and spectroscopic investigations. Spectroscopy grade solvents (ethanol and glycerol) were procured from M/s. S.D Fine Chemicals and used without further purification.

\subsection{Synthesis of citric acid-urea based carbon nanodot: General Procedure}

An intimate mixture of citric acid (2 g, $10.4 \mathrm{mmol})$ and urea $(1.88 \mathrm{~g}, 31.3 \mathrm{mmol})$ was heated to $210^{\circ} \mathrm{C}$ on a heating mantle for 10 min under atmospheric conditions (CND2) and $\mathrm{N}_{2}$ conditions (CND1). The yellow-brown reaction mixture was cooled to room temperature and dissolved in ethanol $(10 \mathrm{ml})$. The ethanolic solution was centrifuged at $10,000 \mathrm{rpm}$ for $10 \mathrm{~min}$. Subsequently, the supernatant was concentrated and purified by column chromatography.

1.2 Synthesis of citric acid-diaminopropane based carbon nanodot under $\mathrm{N}_{2}$ atmosphere (CND3)

A solution of citric acid ( $2 \mathrm{~g}, 10.4 \mathrm{mmol})$ in diaminopropane $(2.5 \mathrm{~mL}, 30 \mathrm{mmol})$ was heated to $210^{\circ} \mathrm{C}$ on a heating mantle for $15 \mathrm{~min}$ under $\mathrm{N}_{2}$ conditions. The reddish brown reaction mixture was cooled to room temperature and dissolved in ethanol $(20 \mathrm{ml})$. The ethanolic solution was centrifuged at $10,000 \mathrm{rpm}$ for $10 \mathrm{~min}$. Subsequently, the supernatant was concentrated and purified by column chromatography.

\section{Experimental Methods}

Ground-state absorption spectra were recorded using a JASCO model V530 spectrophotometer (Tokyo, Japan). Steady-state (SS) fluorescence spectra were recorded using either HITACHI model F-4010 spectrofluorimeter (Tokyo, Japan) or HORIBA FluoroMax-4. The nanosecond fluorescence decays were measured using diode laser ( $374 \mathrm{~nm} \& 445 \mathrm{~nm},<100 \mathrm{ps}, 1$ $\mathrm{MHz}$ and $490 \mathrm{~nm}, \sim 1.3 \mathrm{~ns}, 1 \mathrm{MHz}$ ) based time-correlated single photon counting (TCSPC) setup (IBH, UK). In the present work, a special PMT detector (IBH, UK) was used for the fluorescence decay measurements. The instrument response function for this setup is $\sim 180 \mathrm{ps}$ at FWHM for $374 \&$ $445 \mathrm{~nm}$ excitations. For lifetime measurements, fluorescence decays were recorded at the magic angle $\left(54.7^{\circ}\right)$ with respect to the vertically polarized excitation light. Fluorescence anisotropy studies were carried out by measuring the fluorescence decays/spectra for parallel $\left(\mathrm{I}_{\|}(t)\right)$ and perpendicular $\left(\mathrm{I}_{\perp}(t)\right)$ 
polarizations with respect to the vertically polarized excitation light. The anisotropy decay function, $r(t)$, was constructed using the following relation:

$$
r(t)=\frac{I_{\|}(t)-G I_{\perp}(t)}{I_{\|\|}(t)+2 G I_{\perp}(t)}
$$

where, $G$ is a correction factor for the polarization bias of the detection setup. The $G$ factor was independently determined for both steady-state and time-resolved anisotropy measurements by using the horizontally polarized excitation light and measuring the two perpendicularly polarized fluorescence decays with respect to the excitation polarization. All the experiments were carried out at ambient temperature, $25 \pm 1^{\circ} \mathrm{C}$ unless otherwise mentioned.

FTIR spectra were recorded on Bruker Tensor III using $\mathrm{NaCl}$ cell and neat samples. For transmission electron microscopy (TEM) measurements the dilute solution was dispersed by sonication and $10 \mu \mathrm{L}$ of it was immediately loaded on activated carbon coated grids ( $200 \mathrm{mesh}$ ). The grids were activated by glow discharge under ultraviolet light for one hour. After air drying for 16 hours, the grids were examined under Carl Zeiss Libra $120 \mathrm{kV}$ transmission electron microscope at an accelerating voltage of $120 \mathrm{kV}$. For atomic force microscopy (AFM) measurements sample was similar to TEM except it was loaded on mica plates. AFM images were recorded with Solver P47 from NT-MDT, Russia.

\section{Additional Figures}

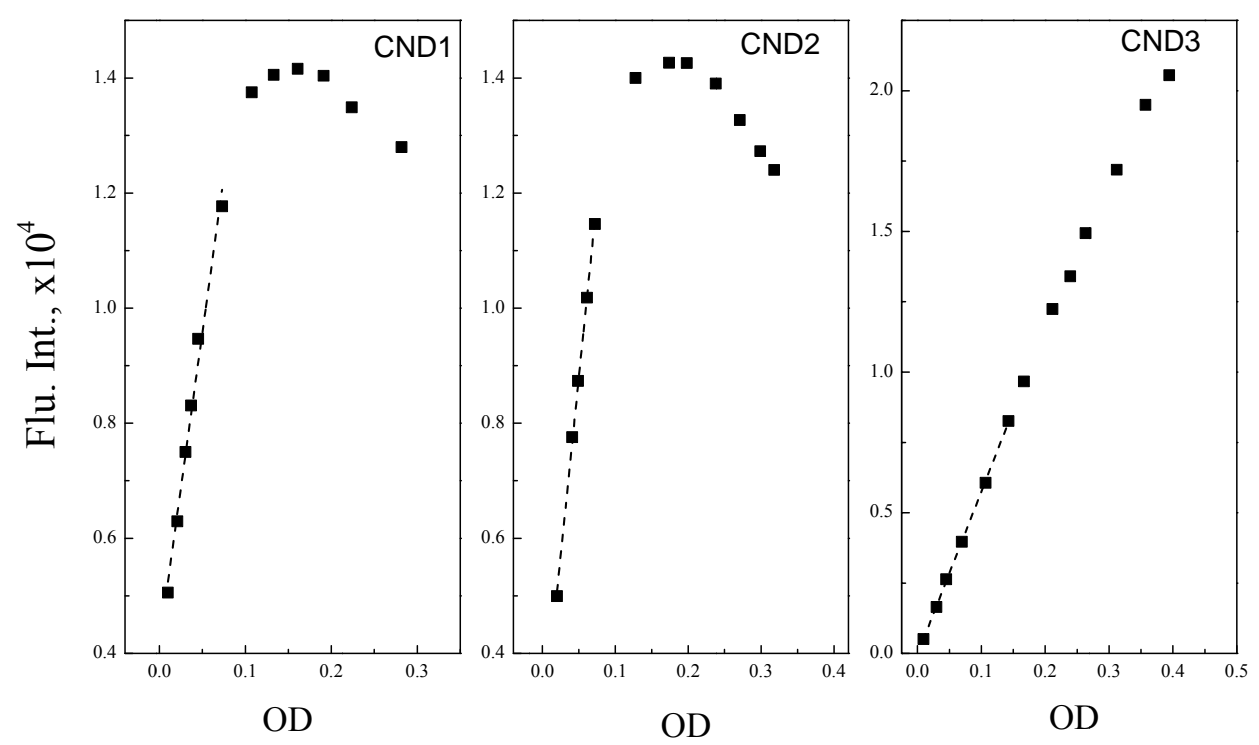

Figure S1. Fluorescence intensity as a function of absorbance. The fluorescence spectral shape remained almost unchanged in the linear OD vs. intensity region. All spectroscopic investigations were carried out with solution OD $<0.1$ (generally $<0.5 \mathrm{mg} / \mathrm{ml}$ ). At very high CND concentrations emission spectra (and also excitation spectra) shows appearance of multiple bands with decrease in intensity for the main emission band in spectral shape. 


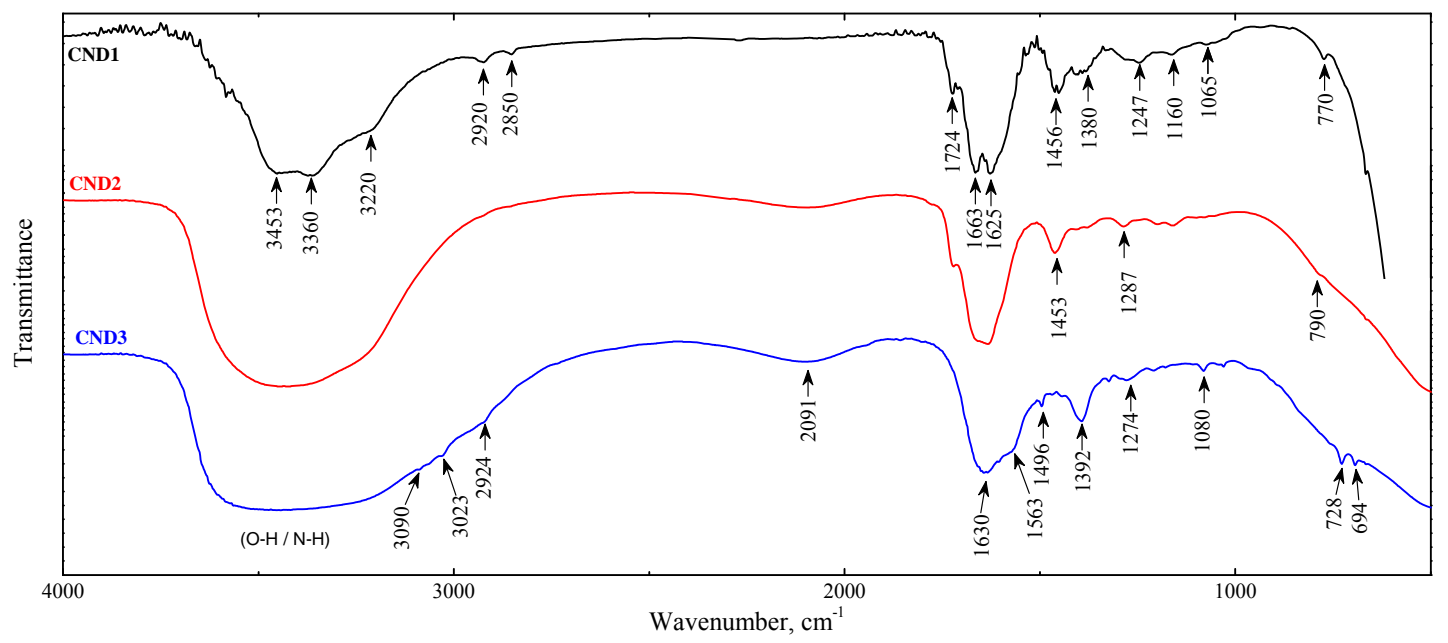

Figure S2. The characteristic absorption bands of surface functional groups in CND are detected by FT-IR spectra. Presence of $-\mathrm{OH}$ and $-\mathrm{NH}$ vibrational bands are evident from the band around 3600$3100 \mathrm{~cm}^{-1}$. The broad bands at 3000 and $2100 \mathrm{~cm}^{-1}$ along with amide bands in the $1650-1400 \mathrm{~cm}^{-1}$ region for CND3 confirms amino-rich functional groups compared to CND1 or CND2. CND3 also shows presence of azide $\mathrm{N}=\mathrm{N}^{+}=\mathrm{N}^{-}$via asymmetric stretch at $2091 \mathrm{~cm}^{-1}$. Small but sharp peak at 1496 $\mathrm{cm}^{-1}$ in CND3 further shows the presence of $\mathrm{NH}_{3}{ }^{+}$for its deformation stretch. The peak at $1392 \mathrm{~cm}^{-1}$ confirms presence of $\mathrm{O}=\mathrm{C}-\mathrm{O}^{-}$symmetric stretching in CND3. Sharp band near $1724 \mathrm{~cm}^{-1}$ (in CND1 \& $\mathrm{CND} 2$ ) is due to $\mathrm{C}=\mathrm{O}$ stretching of aldehyde and ketones, absent in CND3. The overtone band of $\mathrm{C}=\mathrm{O}$ stretching at $3453 \mathrm{~cm}^{-1}$ is clearly evident in CND1. Presence of primary amide is indicated by the $\mathrm{C}=\mathrm{O}$ stretching band at $1663 \mathrm{~cm}^{-1}$ (i.e. Amide I band) in all CND's. Relative abundance of Amide II band at $1563 \mathrm{~cm}^{-1}$ due to $-\mathrm{NH}_{2}$ deformation of primary alkyl amide is evident in CND3. Peak at $1455 \mathrm{~cm}^{-1}$ (CND1, CND2) is due to $-\mathrm{CH}_{2}$ scissor vibrations from $-\mathrm{CH}_{2}$ - aliphatic compounds (very weak in CND3). Bands near $2920 \mathrm{~cm}^{-1}$ in $\mathrm{CND} 1$ come from $-\mathrm{CH}_{2}$ stretching vibrations, showing the nanodots are aliphatic. Presence of unsaturation is visible from the band at $3220 \mathrm{~cm}^{-1}$ (alkyne C-H stretch). Presence of epoxy ethers are shown by C-O stretching vibration near $1275 \mathrm{~cm}^{-1}$. The near $1080 \mathrm{~cm}^{-1}$ band in CND's shows the presence of aliphatic ethers (C-O-C asymmetric stretch). The $1160 \mathrm{~cm}^{-1}$ band in CND1 \& CND2 is for C-O stretching of primary alcohol. Peaks at 728, 790, 694 $\mathrm{cm}^{-1}$ are from various bending vibrations of $-\mathrm{C}-\mathrm{H}$. 

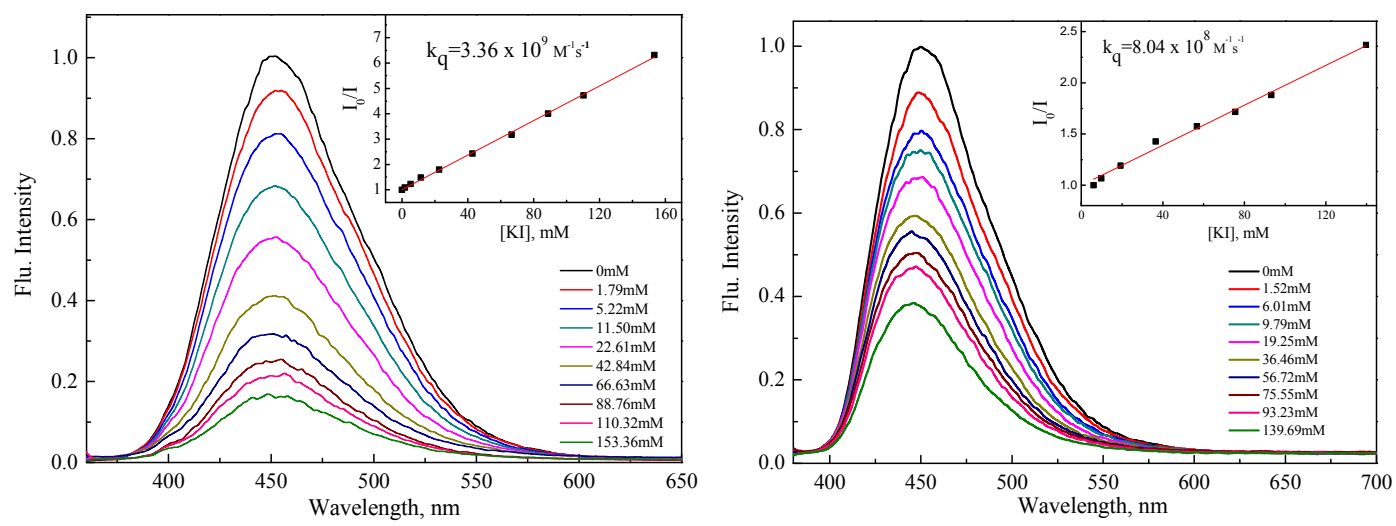

Figure S3. Steady-state fluorescence quenching of CND2 (left) and CND3 (right) by KI in water indicates accessibility of emission centres by quencher.

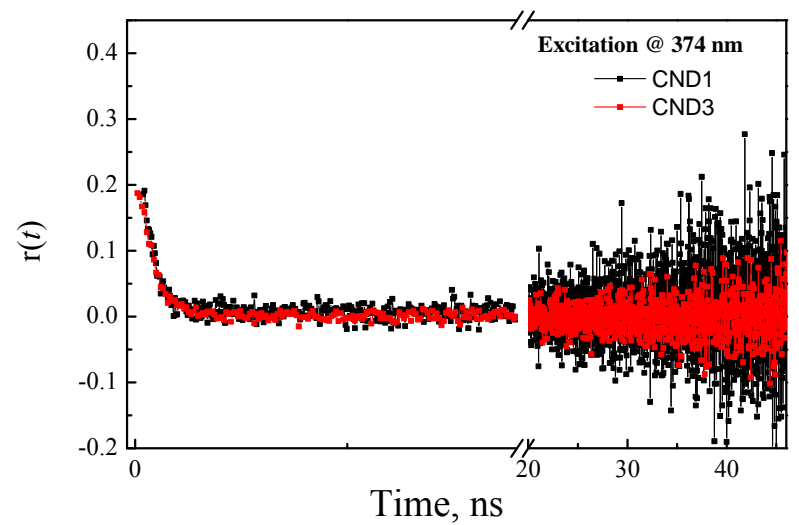

Figure S4. Time-resolved anisotropy decays of CND. Rotational correlation time is around $0.35 \mathrm{~ns}$ obtained from the fitting of initial few nanosecond anisotropy decay trace.

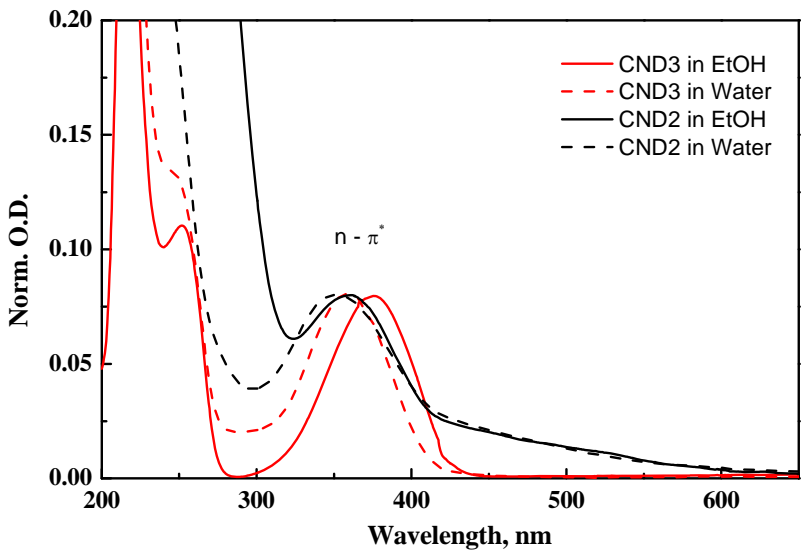

Figure S5. Absorption spectra shows hypsochromic shift with increase in solvent polarity. 

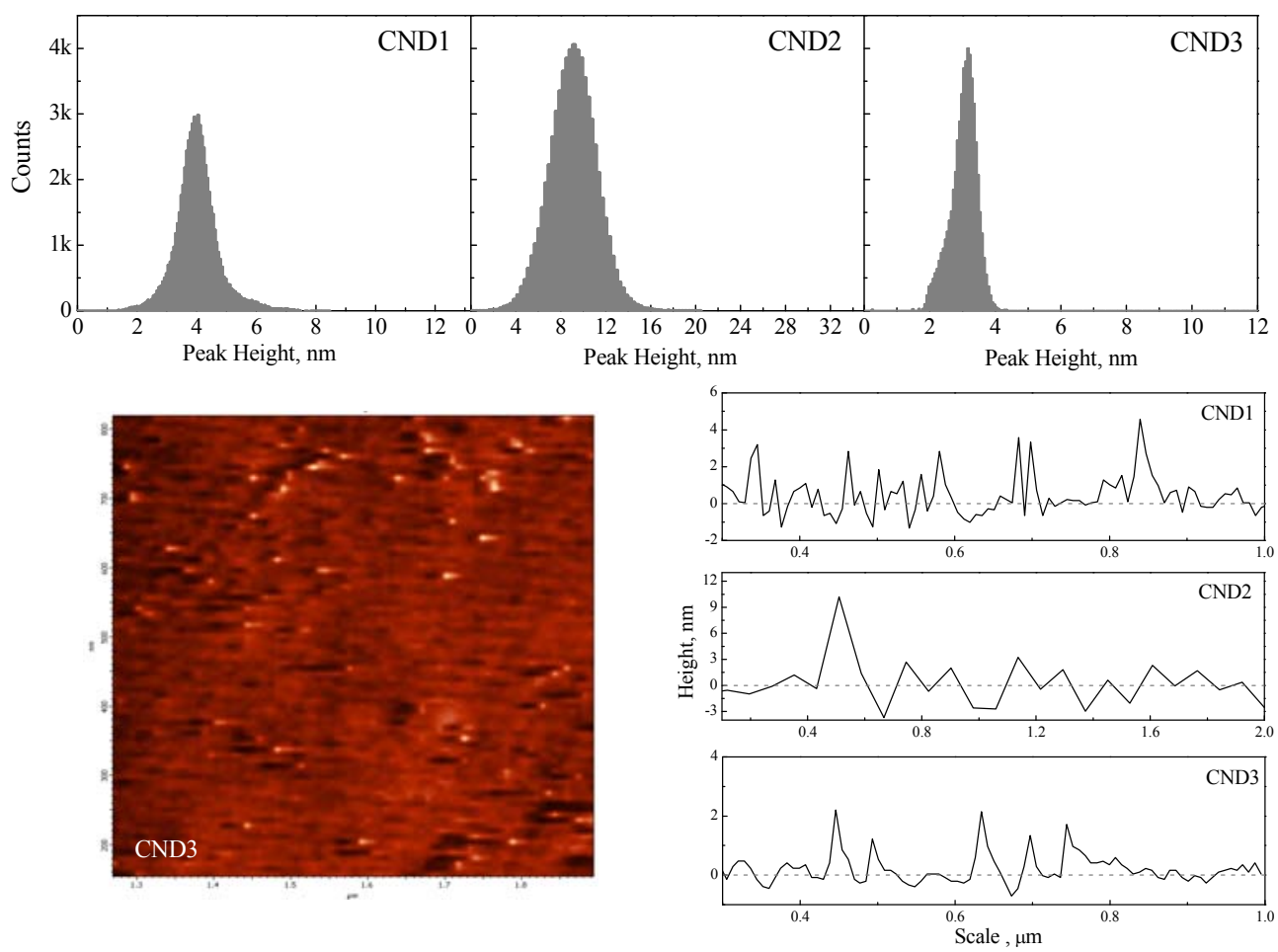

Figure S6. Particle height distribution and particle height traces along with AFM image for CNDs.
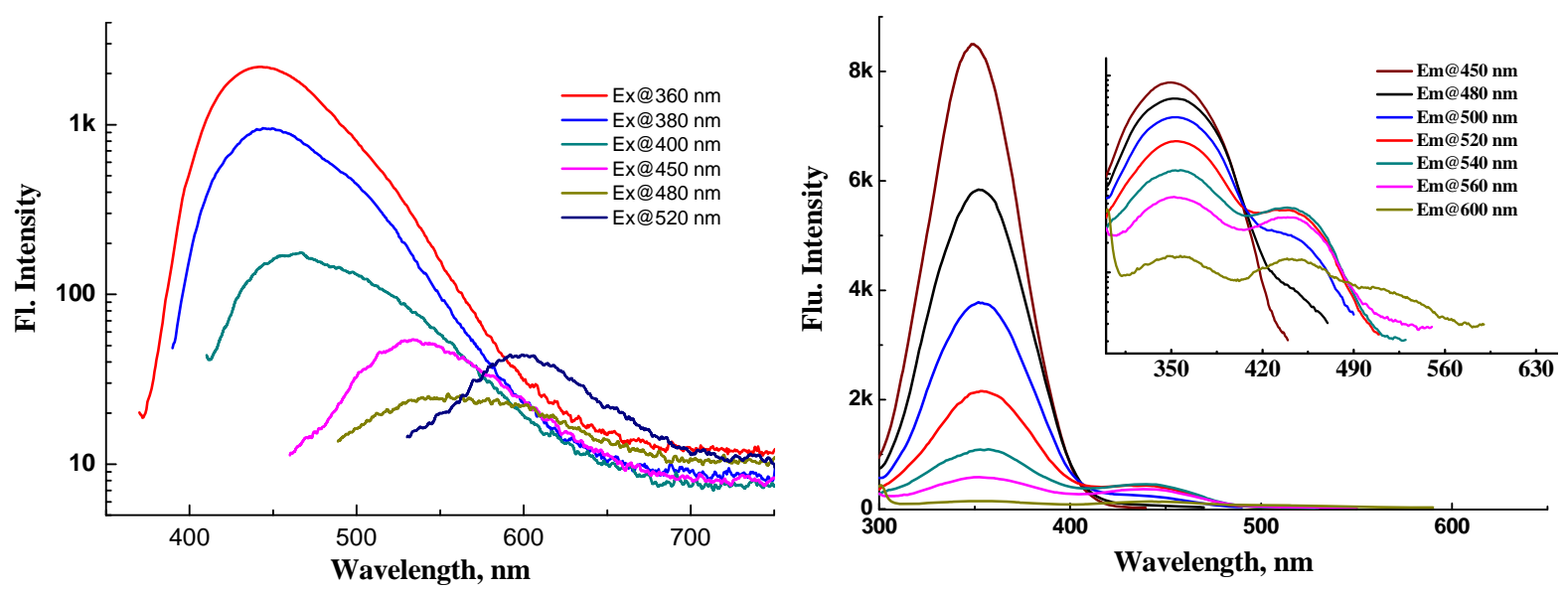

Figure S7. Fluorescence spectra (left) of CND1 in water at different excitation wavelength. The excitation and emission slit widths are increased for recording weak emission spectra at far red excitations. Excitation spectra (right) of the same at different emission wavelengths. Involvement of multiple electronic transitions at different wavelength is clearly evident. 

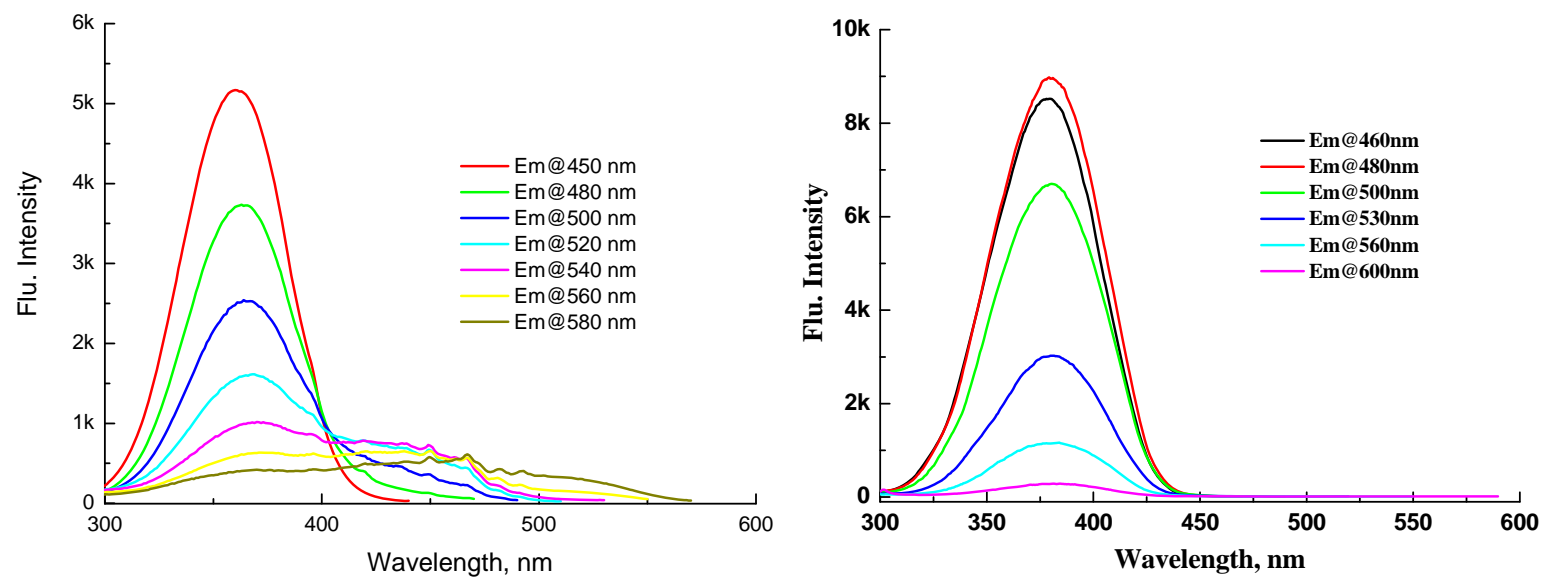

Figure S8. Excitation spectra of CND2 (left) and CND3 (right) in ethanol at different emission wavelengths.
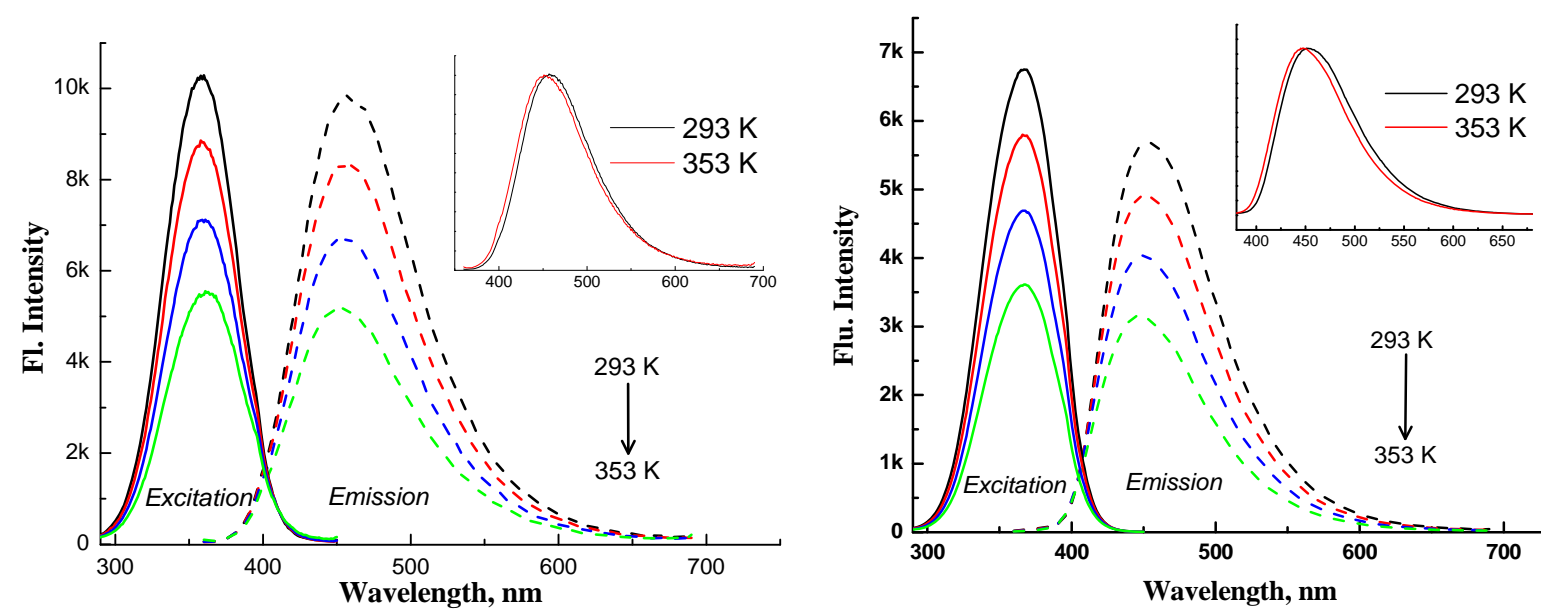

Figure S9. Temperature dependent excitation spectra (solid line, emission at $460 \mathrm{~nm}$ ) and emission spectra (dashed line, excitation at $350 \mathrm{~nm}$ ) of CND2 (left) and CND3 (right) in water. Inset shows normalised emission spectra at $293 \mathrm{~K}$ and $353 \mathrm{~K}$. Blue shift of emission spectra is explained from the differential temperature effect on $\mathrm{H}$ - and J-type aggregates. Small increase in $320 \mathrm{~nm}$ excitation band with concentration (Figure 5b) indicates the classical H-type dimer/aggregate, which shows increase in intensity with increase in temperature. At the far red region, emission has sizeable contribution from J-type dimer/aggregates, which shows significant decrease in intensity with temperature. 

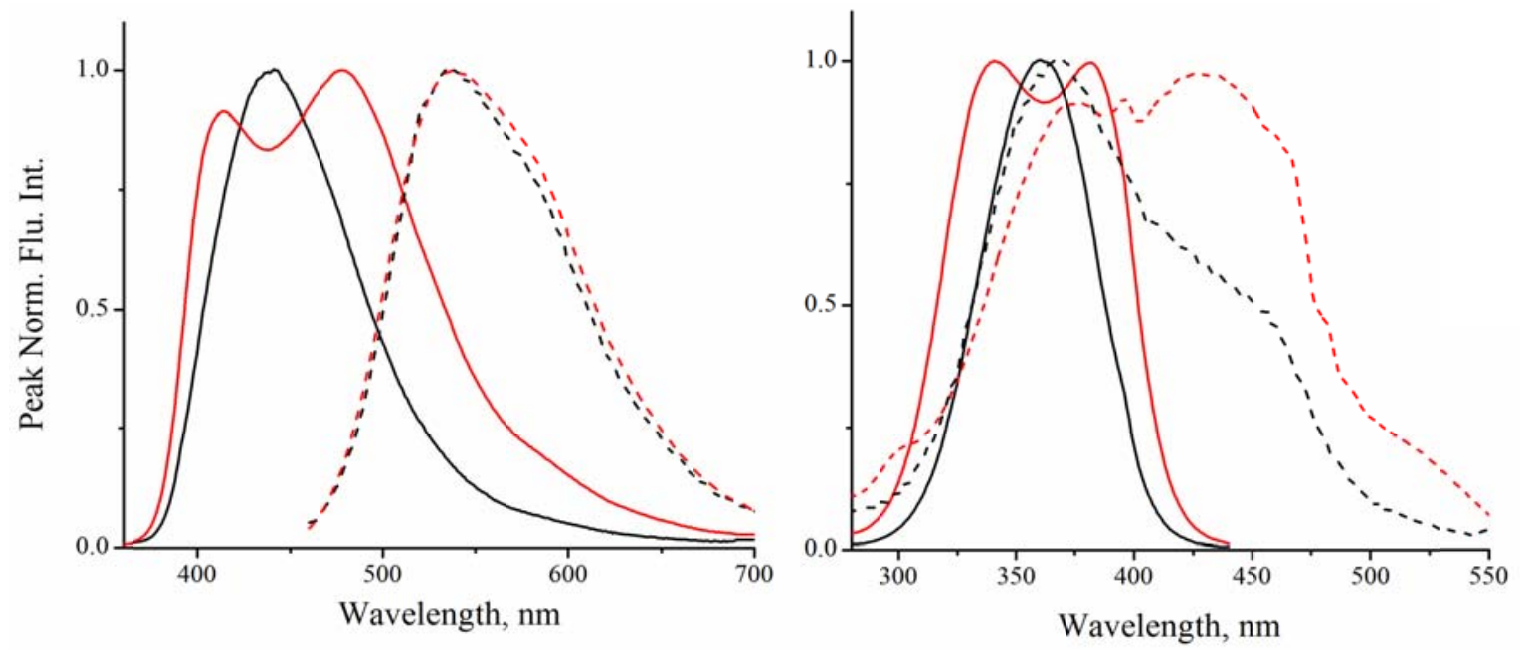

Figure S10. Emission (left) and excitation (right) spectra at very low $(<0.1 \mathrm{mg} / \mathrm{ml}$, black) and high $(>10 \mathrm{mg} / \mathrm{ml}$, red) concentrations in ethanol indicate multiple excitation \& emission band due to aggregates. The emission spectra with 350 and $450 \mathrm{~nm}$ excitations are indicated by solid and dashed lines, respectively. The excitation spectra with emission fixed at 460 and $560 \mathrm{~nm}$ are similar represented by solid and dashed lines, respectively. Appearance of multiple electronic transitions with increase in concentration indicates aggregation behaviour.
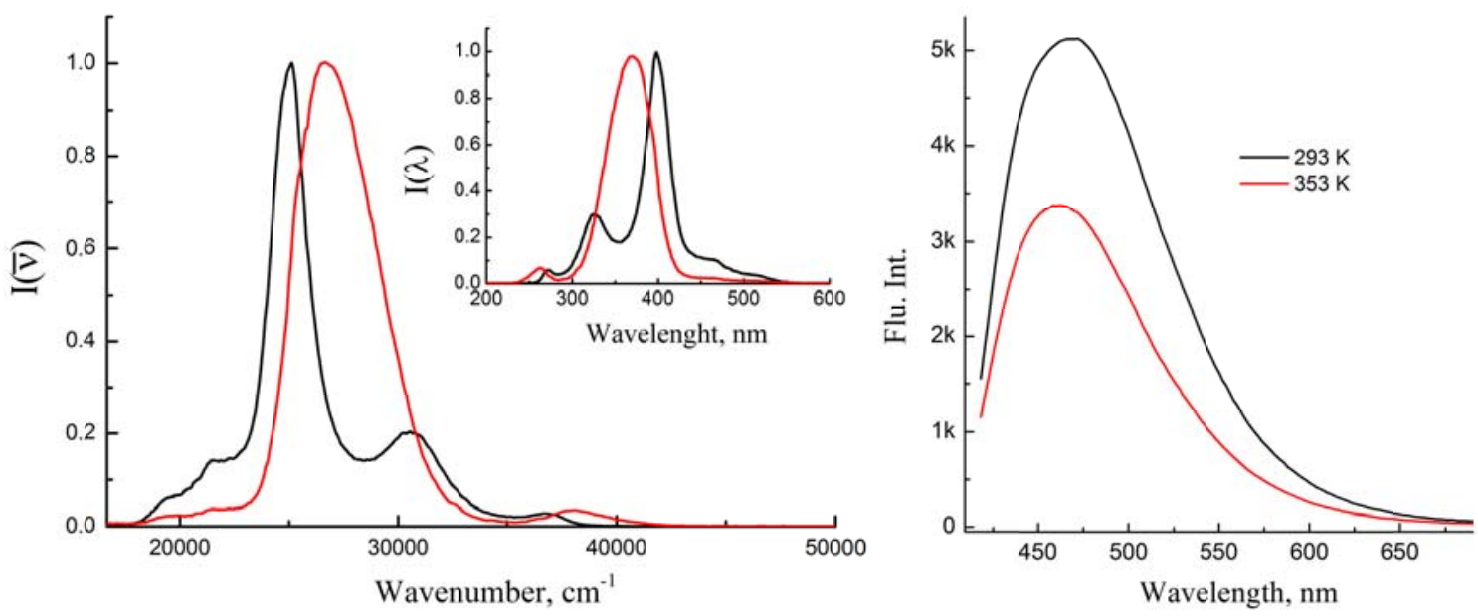

Figure S11. Excitation spectra (left) in wavenumber (same as inset, in wavelength scale) at very low $(<1 \mathrm{mg} / \mathrm{ml}$, red) and very high $(>20 \mathrm{mg} / \mathrm{ml}$, black) CND concentrations in water. The sharp low energy excitation spectra at $25000 \mathrm{~cm}^{-1}$ (corresponding to $400 \mathrm{~nm}$ excitation band) indicate J-type aggregation at higher concentrations. Emission spectra (right) with excitation at $410 \mathrm{~nm}$ at 273 and $353 \mathrm{~K}$. 


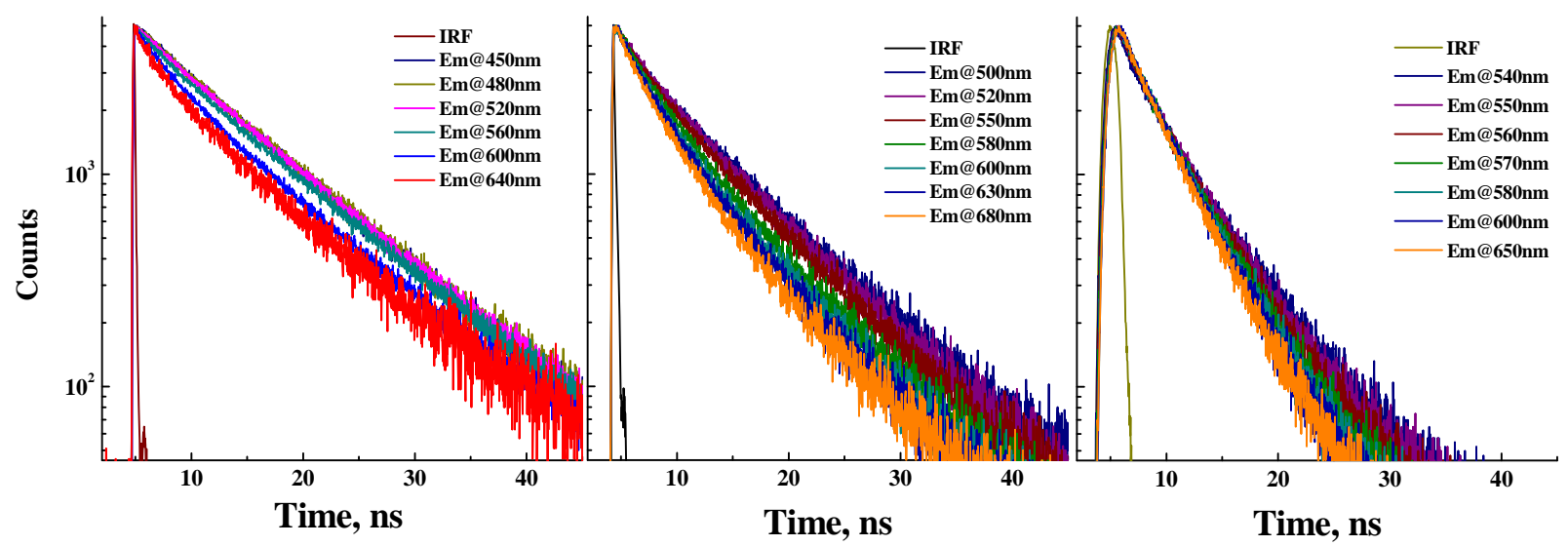

Figure S12. Time resolved fluorescence decay of CND1 in ethanol at different emission wavelengths with excitations at $374 \mathrm{~nm}$ (left), $445 \mathrm{~nm}$ (middle) and $490 \mathrm{~nm}$ (right).
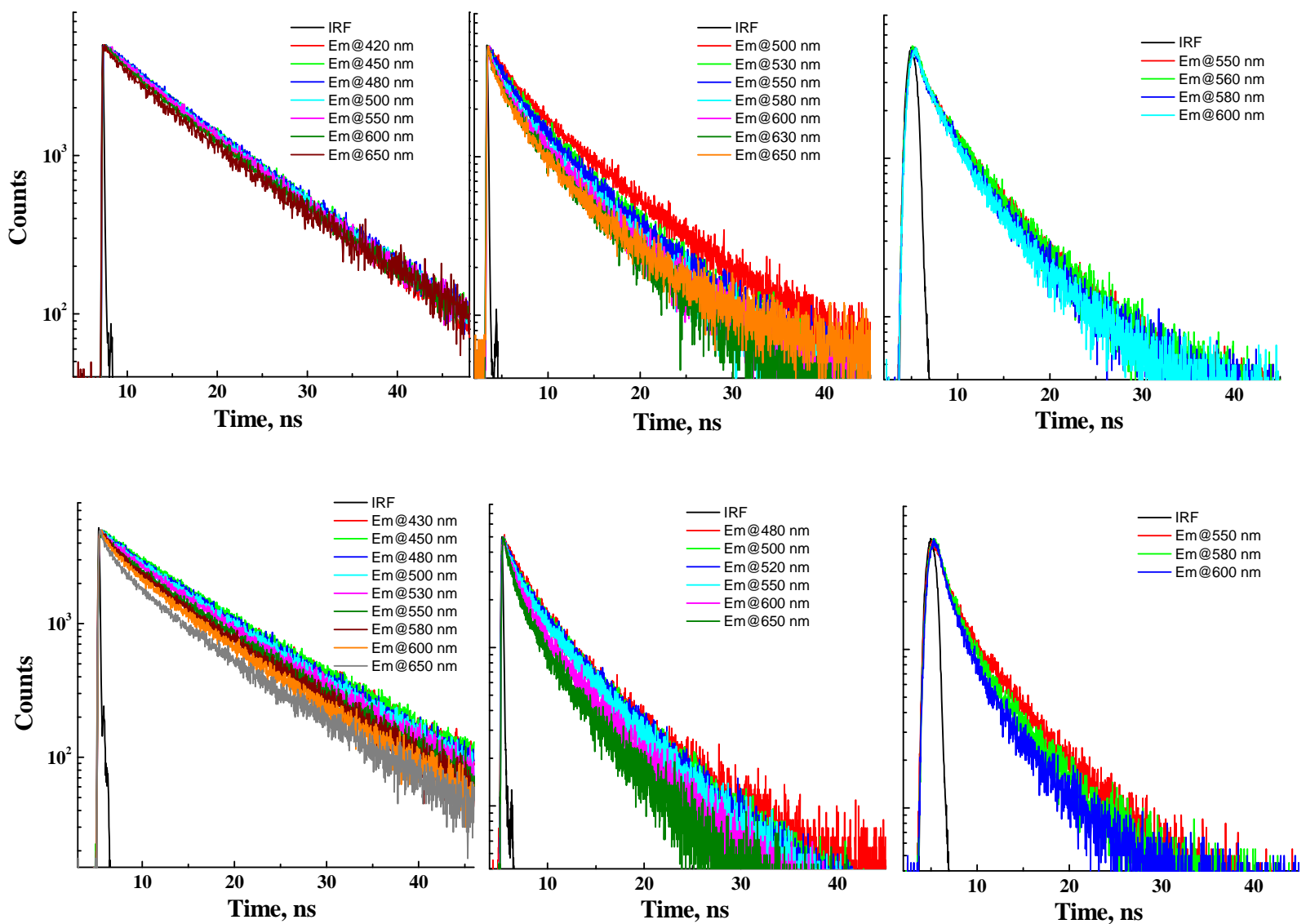

Figure S13. Time resolved fluorescence decay of CND2 in ethanol (top panel) and water (bottom panel) at different emission wavelengths with excitations at $374 \mathrm{~nm}$ (left), $445 \mathrm{~nm}$ (middle) and 490 nm (right). 

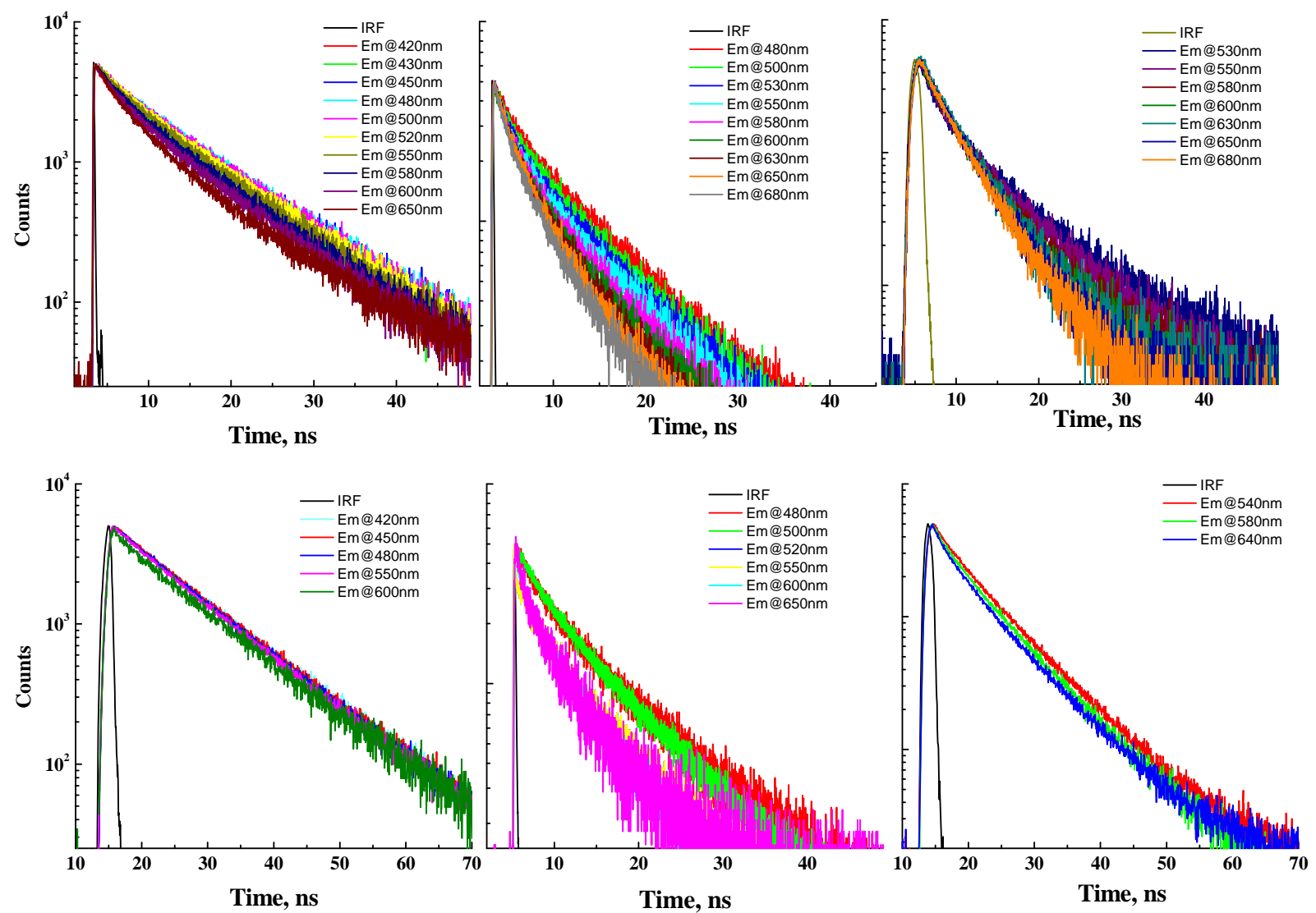

Figure S14. Time resolved fluorescence decay of CND3 in ethanol (top panel) and water (bottom panel) at different emission wavelengths with excitations at $374 \mathrm{~nm}$ (left), $445 \mathrm{~nm}$ (middle) and 490 nm (right).

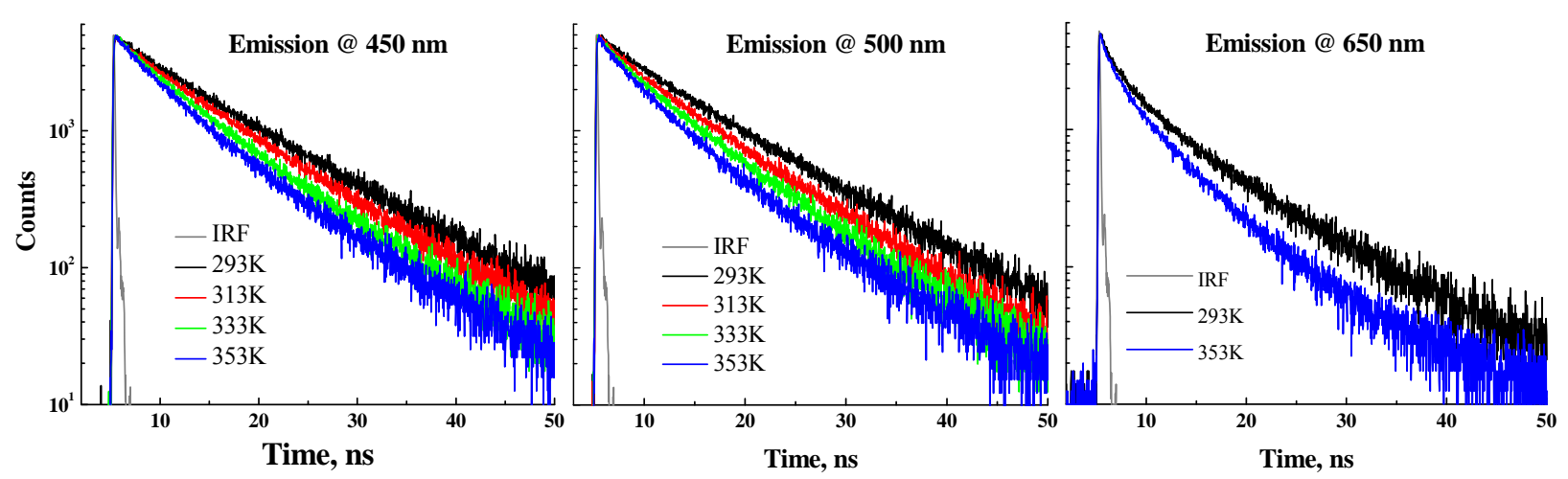

Figure S15. Time resolved fluorescence decay of CND1 in water at different emission wavelengths. Fluorescence decays become faster with increase in temperature all throughout the emission spectrum. 

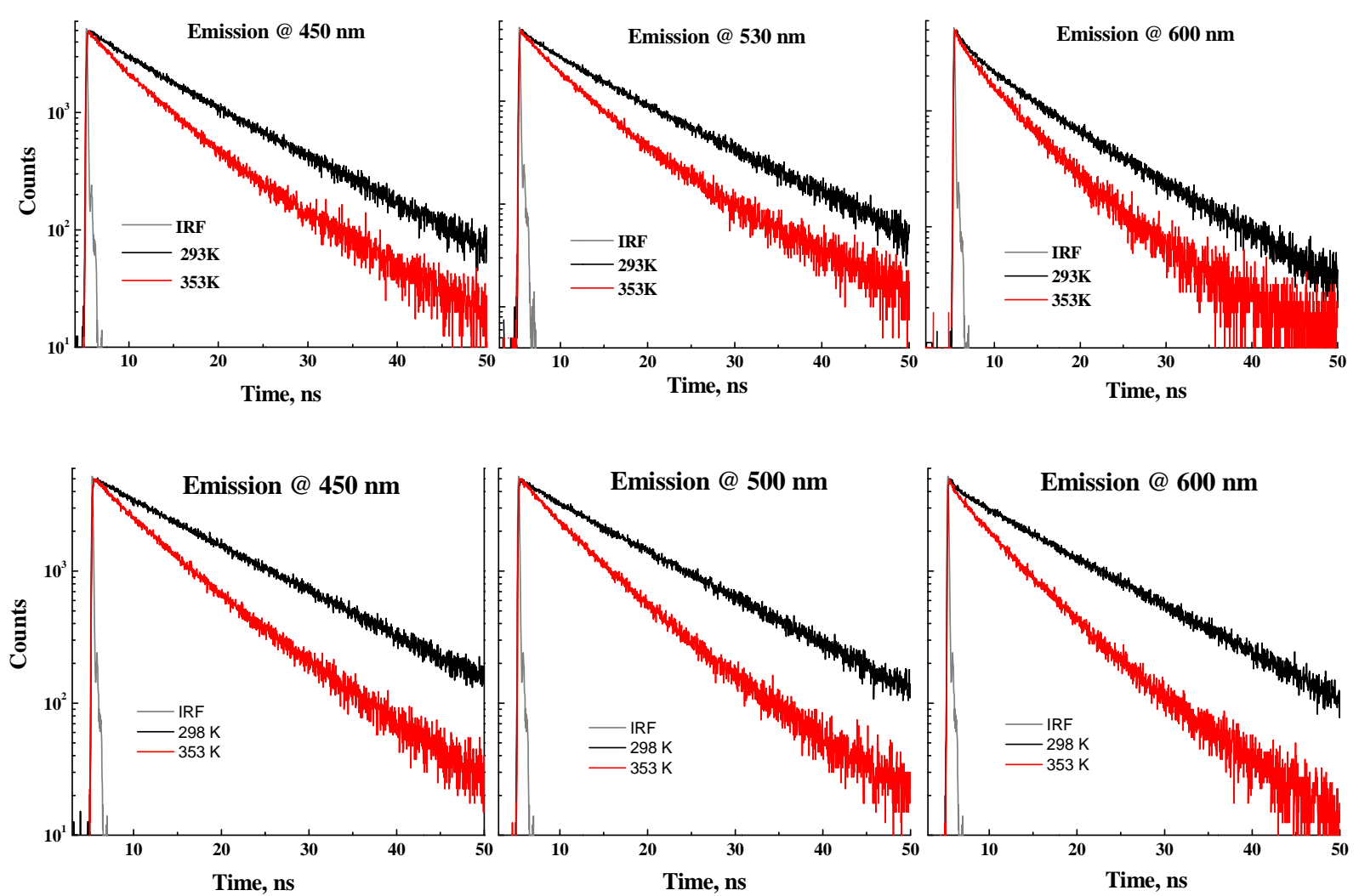

Figure S16. Time resolved fluorescence decay of CND2 (top panel) and CND3 (bottom panel) in water at different emission wavelengths. Fluorescence decays become faster with increase in temperature all throughout the emission spectrum.

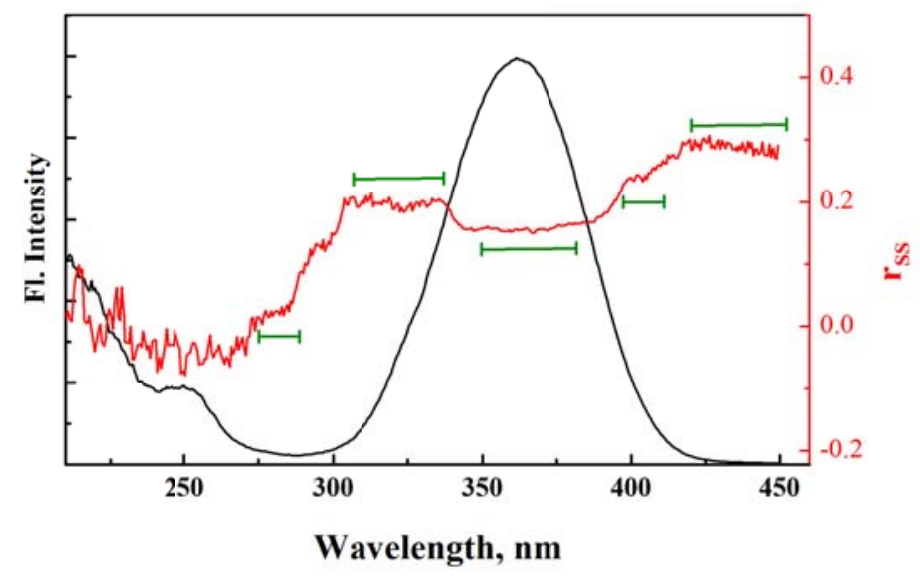

Figure S17. Fluorescence excitation spectra (black line) and excitation anisotropy spectra (red line) of CND1 in glycerol at moderate concentrations for emission at $460 \mathrm{~nm}$ also indicate in-band heterogeneity. 